\title{
La Competencia Parental en las etapas de Educación Infantil y Primaria: Diseño y Validación de un Instrumento
}

\section{Parental Competence in Infant and Primary Education: Design and validation of an instrument}

\author{
María Ángeles Blanco Portillo ${ }^{1}$ \\ Gelesblan@yahoo.es \\ Paz Cánovas Leonhardt \\ Paz.canovas@uv.es \\ Piedad Sahuquillo Mateo \\ Piedad.sahuquillo@uv.es \\ Amparo Pérez Carbonell \\ Amparo.perez@uv.es \\ Verónica Riquelme Soto \\ Veronica.riquelme@uv.es \\ Universidad de Valencia, España
}

\section{Resumen:}

Actualmente, la familia se encuentra ante retos que requieren de su eficiencia y eficacia en la crianza, protección y orientación de los hijos, es decir, la parentalidad y la capacidad de los padres de ejercerla de forma competente. Desde este punto de vista, destaca la necesidad de un modelo educativo centrado en el desarrollo de las competencias parentales que proporcione conocimientos, habilidades y actitudes que optimicen el rol parental respecto al desarrollo pleno y sistémico del ser humano. Ante la necesidad de conocer si los padres asumen de forma óptima su papel en la educación de sus hijos,

\begin{abstract}
:
Currently, families are faced with continuous challenges that require efficiency and effectiveness in the process of upbringing and a conscious awareness of parenthood and how to implement it based on protecting and orienting children. From this point of view, the need for an educational model focused on the development of parental competences, that is, on providing knowledge, skills and attitudes that optimize parental roles with respect to the full and systemic development of human beings becomes evident. Given the need for parents to exercise their role in the education of their children, the main objective
\end{abstract}

1 Dirección para correspondencia (correspondence address):

María Ángeles Blanco Portillo. Dirección: Avd Vicente Andrés Estellés 10 -9b. Alaquàs. 46970, Valencia (España). E-mail: blanco_marpor@gva.es 
La Competencia Parental en las etapas de Educación Infantil y Primaria: Diseño y Validación de un Instrumento

María Ángeles Blanco Portillo, Paz Cánovas Leonhardt, Piedad Sahuquillo Mateo, Amparo Pérez Carbonell y Verónica Riquelme Soto

se plantea como objetivo principal de este artículo el diseño de un instrumento para el análisis de la competencia parental en las etapas de Educación Infantil y Primaria. Para responder a este objetivo realizamos un análisis de la literatura científica y revisamos los principales instrumentos de evaluación, nacionales e internacionales, referidos a este campo objeto de estudio. Posteriormente, procedimos al diseño y validación de contenido y constructo del instrumento a través de comités de expertos y al análisis de fiabilidad factorial exploratorio y confirmatorio. Los resultados obtenidos evidencian que se trata de una herramienta con adecuada validez, discriminante y convergente, para la población objeto de estudio; sin embargo, se observa una baja fiabilidad para algunos de los factores/dimensiones.

\section{Palabras clave:}

Educación parental; responsividad parental; competencia parental; instrumento de medida. of this article is the design of an ad hoc instrument for the analysis of parental competence in Infant and Primary Education. In order to respond to this objective, we conducted an analysis of the scientific literature and reviewed some of the existing national and international evaluation instruments related to this field. In addition, we designed an instrument and undertook a study of content and construct validation through expert committees and an exploratory and confirmatory factorial reliability analysis. Results evidence that this is a test that presents an adequate discriminant and convergent validity for the population under study but a low reliability for some of the factors/dimensions.

\section{Key words:}

Parenting education; parent responsivity, parental competence; measuring instrument.

\section{Resumé:}

Actuellement, la famille est confrontée à des défis qui exigent son efficacité dans l'éducation, la protection et l'orientation des enfants, c'est-à-dire le rôle parental et la capacité des parents à l'exercer avec compétence. De ce point de vue, il est nécessaire de disposer d'un modèle éducatif axé sur le développement des compétences parentales qui fournisse des connaissances, des aptitudes et des attitudes permettant d'optimiser le rôle parental en ce qui concerne le développement complet et systémique de l'être humain. Étant donné la nécessité de savoir si les parents assument leur rôle dans l'éducation de leurs enfants de manière optimale, l'objectif principal de cet article est la conception d'un instrument pour l'analyse de la compétence parentale dans les étapes de la petite enfance et de l'enseignement primaire. Afin de répondre à cet objectif, nous avons effectué une analyse de la littérature scientifique et passé en revue les principaux instruments d'évaluation nationaux et internationaux relatifs au domaine étudié. Ensuite, nous avons procédé à la conception et à la validation du contenu et de la construction de l'instrument par le biais de comités d'experts et d'une analyse de fiabilité factorielle exploratoire et confirmatoire. Les résultats obtenus montrent qu'il s'agit d'un outil ayant une validité discriminante et convergente adéquate pour la population étudiée ; cependant, une faible fiabilité est observée pour certains des facteurs/dimensions.

\section{Mots clés:}

Éducation parentale; réactivité parentale; compétence parentale; instrument de mesure.

Fecha de recepción: 16-10-2019

Fecha de aceptación: 17-03-2020 
María Ángeles Blanco Portillo, Paz Cánovas Leonhardt, Piedad Sahuquillo Mateo, Amparo Pérez Carbonell y Verónica Riquelme Soto

\section{Introducción}

Gran parte de los estudios realizados en el ámbito familiar se han centrado en determinar qué estilos educativos propiciaban un desarrollo óptimo e integral del menor así como sus consecuencias en el niño. Sin embargo, esta perspectiva nos proporciona una visión bastante reducida de la parentalidad competente, ya que la competencia va más allá, entendida, pues, como la capacidad de poner en marcha al mismo tiempo y de forma interrelacionada los conocimientos, capacidades, habilidades y actitudes necesarias para solventar las tareas o situaciones con un cierto nivel de calidad y eficacia (Bisquerra y Pérez, 2007; Zabala y Arn$\mathrm{au}, 2007$ ), donde prime la singularidad del menor, se reconozcan sus necesidades y se acepte su individualidad como sujeto único e irrepetible. En la satisfacción de dichas necesidades surge la competencia parental y, por tanto, las dimensiones que la integran. Todas suponen la detección y satisfacción de las mismas, dado que si los progenitores son capaces de detectarlas pero no de satisfacerlas estarían incurriendo igualmente en la incompetencia parental.

Desde nuestra perspectiva, la competencia parental se entiende como "el conjunto de capacidades que permiten a los padres afrontar de modo adaptativo la tarea vital que han de asumir" (Cánovas y Sahuquillo, 2011, p. 6) de modo que contribuyan al buen trato hacia los menores y prime su interés superior.

Al respecto, compartimos los estudios realizados, entre otros, por Masten y Curtis, 2000; Pereda y Berrocal, 2001; Repetto, 2003; Roe, 2003; Tejada y Navío, 2004; Barudy y Dantagnan, 2005, 2010; Tejada, 2005; Masten y Obradovic, 2006; Repetto, Pena, Mudarra y Uribarri, 2007; Rutter, 2007; Rodrigo et al., 2008; Rodrigo, Martín, Cabrera y Máiquez, 2009. Sin embargo, consideramos necesario integrar dos dimensiones no contempladas como tal en estudios previos como son el apego y el ejercicio parental desde la responsividad. Entendida como la tendencia del adulto responsable de la crianza en reconocer señales conductuales del niño y en relación a ellas proporcionar una respuesta adecuada y equilibrada (Wilcox, Kauni y Caswell, 1990). Requiere la sensibilidad de los adultos para identificar e interpretar las conductas con el fin de ajustar la respuesta al contexto e individualidad del menor desde el afecto y no desde la propia satisfacción de necesidades.

Así pues, a continuación, en la tabla 1, presentamos de forma sinteti- 
La Competencia Parental en las etapas de Educación Infantil y Primaria: Diseño y

Validación de un Instrumento

María Ángeles Blanco Portillo, Paz Cánovas Leonhardt, Piedad Sahuquillo Mateo,

Amparo Pérez Carbonell y Verónica Riquelme Soto

zada las dimensiones y subdimensiones que conforman para nosotras la competencia parental.

Tabla 1

Dimensiones y subdimensiones de la competencia parental

\begin{tabular}{|c|c|}
\hline DIMENSIÓN & SUBDIMENSIÓN \\
\hline \multirow[t]{3}{*}{ Cuidado y protección } & Atención médica \\
\hline & Salud \\
\hline & Protección de riesgos \\
\hline \multirow[t]{8}{*}{ Relación educativa } & Identidad \\
\hline & Autonomía \\
\hline & Transmisión de valores \\
\hline & Comunicación \\
\hline & Autoridad \\
\hline & Apoyos y reconocimiento \\
\hline & Expectativas \\
\hline & Familia-escuela \\
\hline \multirow[t]{7}{*}{ Socio-afectiva y de apego } & Desarrollo Socio-afectivo \\
\hline & Comunicación afectiva \\
\hline & Resolución de conflictos \\
\hline & Habilidades de y para la vida \\
\hline & Contexto emocional \\
\hline & Apego seguro y protección \\
\hline & Resiliencia \\
\hline \multirow[t]{7}{*}{ Adaptativa y responsividad parental } & Aceptación y reconocimiento \\
\hline & Flexibilidad \\
\hline & Cambios normativos y paranormativos \\
\hline & Autopercepción del rol parental \\
\hline & Eficiencia económica \\
\hline & Satisfacción personal \\
\hline & Acuerdos familiares \\
\hline
\end{tabular}

Fuente: Blanco (2016).

En el estudio de las competencias parentales, como campo emergente, se evidencia a partir de la búsqueda realizada en bases de datos especializadas (TESEO REDINET, Eurydice, ISOC, ERIC, SCOPUS, ICYT, DATRI, , Redinat) que son muy pocas las pruebas e instrumentos existentes si las comparamos con las que se utilizan para medir los estilos de comunicación parental, la implicación de padres y madres en los proce- 
sos educativos, la adaptabilidad de la infancia-adolescencia a diversos contextos, la disciplina, los estilos educativos, etc.

Por otro lado, frecuentemente, estos estudios ponen mayor atención en las debilidades y carencias que presentan padres y madres, sin reparar en la importancia de las relaciones positivas y significativas, las expectativas y las capacidades y/o competencias que les son propias.

Además, cabe añadir, que muchos de los instrumentos quedan obsoletos, dado que no abarca al ser humano desde diferentes dimensiones (afectiva, social, educativa, cognitiva) y un gran número de ellos se centran en las debilidades de los progenitores y el nivel de riesgo, obviando indicadores de protección en la educación familiar que hace que se minimicen los riesgos. Junto a esto, diferentes autores internacionales coinciden en señalar que los instrumentos existentes, aplicados en el ámbito de la psicología clínica, evidencia cierto grado de insuficiencia y limitaciones al ser adaptados a nuestro contexto, tanto a nivel psicométrico como metodológico (Bayot, Hernández y De Julián, 2005; Vázquez, Molina, Ramos y Artazcoz, 2016). Del mismo modo, cabe señalar que, en la mayoría de casos, las herramientas con que se trabaja se enmarcan en planteamientos unidireccionales y, además, se constata la carencia de pruebas que trabajen la competencia parental a partir de situaciones específicas y contextos concretos. Así pues, se hace necesario un enfoque más global y actualizado que integra aspectos tan relevantes como la responsividad y adaptación al rol parental, yendo más allá de la mera detección y respuesta a necesidades.

A partir de un análisis crítico de la revisión de los instrumentos evidenciamos la necesidad de plantear el diseño de un instrumento que parta desde el constructo teórico defendido en líneas anteriores. El objetivo principal de este artículo es el diseño de un instrumento ad hoc para el análisis de la competencia parental en las etapas de Educación Infantil y Primaria.

Para llevar a cabo este objetivo hemos realizado una revisión de la literatura científica existente y analizado los diferentes instrumentos que valoran aspectos de la competencia parental, con el fin de diseñar un instrumento que se adecue a lo que desde nuestra aportación supone y significa competencia parental. 
La Competencia Parental en las etapas de Educación Infantil y Primaria: Diseño y Validación de un Instrumento

María Ángeles Blanco Portillo, Paz Cánovas Leonhardt, Piedad Sahuquillo Mateo, Amparo Pérez Carbonell y Verónica Riquelme Soto

\section{Método}

El estudio se ha basado en una metodología cuantitativa con un diseño transversal, debido al carácter dinámico que tiene el contexto familiar y la individualidad del sujeto. El muestreo ha sido no probabilístico y se centra en el proceso de validación de contenido y de constructo con todo el trabajo de revisión y valoración del grupo de expertos junto con el proceso del análisis métrico de la escala que se aporta como propuesta.

\section{Muestra}

Para realizar el estudio hemos seleccionado tres centros educativos de diferente titularidad (público y concertado) de Educación Infantil y Primaria de la provincia de Valencia. La muestra se compone por un total de 924 familias. Sin embargo, es de interés señalar que el cuestionario fue cumplimentado adecuadamente por un total de 414 familias, tal y como se expone en la tabla 2.

Tabla 2

Muestra del estudio

\begin{tabular}{lccc}
\hline CENTRO & N Total & N Real & N \\
(no responde o se descarta)
\end{tabular}

Fuente: Blanco (2016).

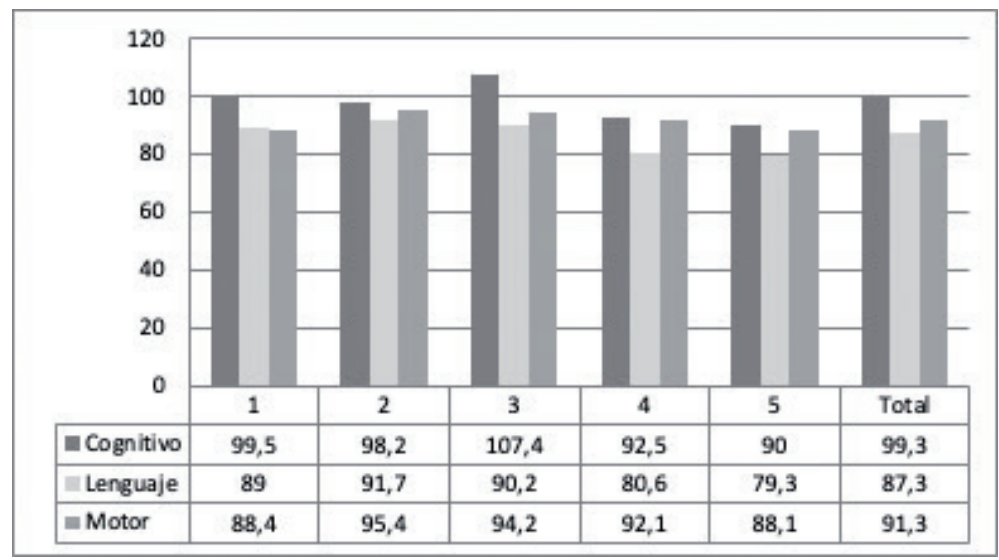

Figura 1. Distribución porcentual de la muestra según el centro. 
María Ángeles Blanco Portillo, Paz Cánovas Leonhardt, Piedad Sahuquillo Mateo, Amparo Pérez Carbonell y Verónica Riquelme Soto

\section{Análisis métrico}

En el proceso de construcción del instrumento nos ceñimos a un diseño de validación de pruebas (Gronlund y Linn, 1990; Jornet, Suárez y Pérez, 2000). Asimismo, contamos con las opiniones, ideas y la experiencia aportada por diferentes agentes: profesorado y personal especializado en temas relacionados con familia. Posteriormente, estas personas pasaron a formar parte del grupo de 13 expertos.

En un primer momento, nuestro instrumento estaba formado por 99 ítems que se agrupaban en dimensiones2 y subdimensiones3. A partir de dicha propuesta, se solicitó a los jueces expertos que analizaran la adecuación y validez tomando en consideración los criterios que a continuación se detallan:

- Univocidad: exactitud a la hora de formular el ítem; sólo es posible comprender cada ítem de un modo concreto.

- Ubicación: se valora si es correcta la asignación de cada ítem a la dimensión en que se enmarque.

- Inteligibilidad: comprensión de cada ítem por parte de las personas a las que se dirige.

- Validez: adecuación del ítem al objeto de estudio.

Con el fin de orientar la labor de los expertos, se les facilitó una escala de valoración: 1 significaba nada, 2 poco, 3 bastante, 4 mucho y 5 totalmente. Tras recoger las valoraciones se realizó un análisis para cada criterio de los señalados anteriormente.

\section{Validez de contenido}

Para analizar este aspecto realizamos dos tipos de análisis. Por una parte, hallamos estadísticos descriptivos de cada uno de los ítems donde calculamos el valor mínimo, máximo, la media y desviación típica y, por otra parte, se halló el coeficiente de variación, para comprobar el grado de variabilidad de las respuestas.

Así, se estimó necesario analizar las puntaciones medias obtenidas, a fin de determinar si los ítems con las valoraciones más altas por parte de los expertos formarían parte del instrumento, puesto que aquellas que

2 Entendemos por dimensión los aspectos centrales de la competencia parental.

3 Entendemos por subdimensión los aspectos que integran cada dimensión. 
asumían puntuaciones bajas serían o bien modificadas o eliminadas del mismo.

De este modo, en función de la escala de valoración de los expertos, los ítems con una puntuación entre 4 ó 5, dentro de los valores estimados para cada criterio a evaluar, se mantenían tal y como habían sido redactados, a la vez que dentro de la dimensión y subdimensión en la que se encontraban. Los ítems con un valor entre 3,70 y 3,99 fueron modificados, atendiendo a cada criterio, mientras que los ítems que tenían una media inferior a 3,70 fueron eliminados. Asimismo, también se suprimieron los ítems que tenían medias superiores a 3,70 pero que su coeficiente de variación era superior a $31 \%$, debido a la falta de homogeneidad en la respuesta de los expertos.

\section{Depuración de los ítems}

Tras calcular el coeficiente de variación y la media de los 99 ítems analizados en relación con la univocidad, ubicación, inteligibilidad y validez, se procedió a depurar el instrumento. De este modo, se reestructuró la prueba inicial (se eliminaron 26 ítems y reformularon 6). El instrumento definitivo4 quedó configurado por 69 preguntas:

- Preguntas referidas a características sociodemográficas relacionadas con la figura parental encuestada y relativas al menor (integra los ítems del 1 al 9, ambos inclusive).

- Preguntas referidas al menor, respecto a su cuidado diario, necesidades educativas especiales, problemas de salud, estado emocional del niño, autonomía, ejercicio físico, higiene y alimentación (integra los ítems del 10 al 17, ambos inclusive).

A su vez, también integra diferentes ítems referentes a las dimensiones o áreas presentadas en la tabla 1. La primera dimensión, referida a las necesidades de Cuidado y protección (atención médica, salud y protección de riesgos), cuenta con un total de 4 ítems; la segunda dimensión, Relación educativa (identidad, autonomía, transmisión de valores, comunicación, autoridad, apoyos y reconocimiento, expectativas y relación familia-escuela), consta de un total de 18 ítems; la tercera dimensión Socioafectiva y apego (desarrollo socioafectivo, comunicación afectiva, resolución de conflictos, habilidades de y para la vida, contex-

4 Se puede consultar el cuestionario en el siguiente enlace: https://bit.ly/2ODn2M6 
to emocional, apego seguro y protección y resiliencia) integra un total de 15 ítems y la cuarta dimensión Adaptativa y responsividad parental (aceptación y reconocimiento, flexibilidad y adaptación a cambios normativos y paranormativos, autopercepción del rol parental, eficiencia económica, satisfacción personal y acuerdos familiares) consta de un total de 13 ítems.

Por último, se incluye una pregunta que alude a los valores que consideran más importante transmitir a sus hijos (ítem 40) y se plantean diferentes situaciones donde el progenitor debe responder señalando la opción que más se ajuste a su estilo educativo (ítem 69).

\section{Análisis de fiabilidad de la Escala de Competencia Parental}

A continuación, se analiza la fiabilidad de esta escala a partir de la interpretación del coeficiente del alfa de Cronbach y la correlación ítem-total. El coeficiente de alfa mide hasta qué punto el ítem planteado contribuye a la medición del rasgo que se pretende medir (Díaz, 1993). En este sentido, George y Mallery (1995) establecen que por debajo de 0,5 el alfa de Cronbach muestra un nivel de fiabilidad no aceptable, un valor entre 0,5 y 0,6 se podría considerar pobre, entre 0,6 y 0,7 sería un nivel cuestionable, entre 0,7 y 0,8 aceptable, entre 0,8 y 0,9 bueno y un valor superior a 0,9 sería excelente. Siguiendo la clasificación de dichos autores se puede afirmar que la fiabilidad es muy alta, con un valor de 0,914.

Cuando observamos la correlación de cada ítem con el total (Coeficiente de Homogeneidad) estamos comprobando si el ítem tiene la misma tendencia de medida que el total de la Escala. De los datos obtenidos (ver tabla 3), se puede observar que la mayoría de los ítems presentan una correlación ítem-total aceptable y su eliminación no implica cambios significativos en la fiabilidad global de la escala.

No obstante, se decidió eliminar del análisis los siguientes ítems por presentar una correlación ítem-total reducida $(<0,3)$ o contribuir al incremento del alfa de Cronbach, ítem: 18, 19, 39, 41, 52, 62, 64 y 68. De este modo, la escala de competencia parental definitiva quedará compuesta por 42 ítems que serán sometidos a un análisis factorial exploratorio y confirmatorio para contrastar la validez de la escala. 
La Competencia Parental en las etapas de Educación Infantil y Primaria: Diseño y Validación de un Instrumento

María Ángeles Blanco Portillo, Paz Cánovas Leonhardt, Piedad Sahuquillo Mateo,

amparo Pérez Carbonell y Verónica Riquelme Soto

Tabla 3

Análisis de fiabilidad general de la escala de competencia parental

\begin{tabular}{|c|c|c|c|c|}
\hline & $\begin{array}{c}\text { Media de } \\
\text { escala si el } \\
\text { elemento se } \\
\text { ha suprimido }\end{array}$ & $\begin{array}{c}\text { Varianza de } \\
\text { escala si el } \\
\text { elemento se ha } \\
\text { suprimido }\end{array}$ & $\begin{array}{l}\text { Correlación } \\
\text { total de } \\
\text { elementos } \\
\text { corregida }\end{array}$ & $\begin{array}{c}\text { Alfa de } \\
\text { Cronbach si el } \\
\text { elemento se } \\
\text { ha suprimido }\end{array}$ \\
\hline Ítem 18 & 224.45 & 180.44 & .138 & .914 \\
\hline Ítem 19 & 224.48 & 179.56 & .243 & .914 \\
\hline Ítem 20 & 224.56 & 176.93 & .422 & .913 \\
\hline Ítem 21 & 224.50 & 178.18 & .370 & .913 \\
\hline Ítem 22 & 224.70 & 175.41 & .423 & .913 \\
\hline Ítem 23 & 224.98 & 172.75 & .466 & .912 \\
\hline Ítem 24 & 224.83 & 172.86 & .514 & .912 \\
\hline Ítem 25 & 224.98 & 172.82 & .431 & .912 \\
\hline Ítem 26 & 224.92 & 174.18 & .385 & .913 \\
\hline Ítem 27 & 224.79 & 175.57 & .356 & .913 \\
\hline Ítem 28 & 224.58 & 176.26 & .451 & .913 \\
\hline Ítem 29 & 225.00 & 170.94 & .504 & .912 \\
\hline Ítem 30 & 224.75 & 173.14 & .543 & .912 \\
\hline Ítem 31 & 224.71 & 174.41 & .449 & .912 \\
\hline Ítem 32 & 224.76 & 174.63 & .471 & .912 \\
\hline Ítem 33 & 224.65 & 175.33 & .433 & .913 \\
\hline Ítem 34 & 224.69 & 173.59 & .554 & .912 \\
\hline Ítem 35 & 224.54 & 176.70 & .477 & .913 \\
\hline Ítem 36 & 224.68 & 175.66 & .388 & .913 \\
\hline Ítem 37 & 225.02 & 172.70 & .369 & .913 \\
\hline Ítem 38 & 224.94 & 174.20 & .324 & .914 \\
\hline Ítem 39 & 224.79 & 175.15 & .280 & .914 \\
\hline Ítem 41 & 224.96 & 176.59 & .229 & .915 \\
\hline Ítem 42 & 225.00 & 173.65 & .380 & .913 \\
\hline Ítem 43 & 224.78 & 175.55 & .337 & .913 \\
\hline Ítem 44 & 224.68 & 174.89 & .485 & .912 \\
\hline Ítem 45 & 224.61 & 176.03 & .436 & .913 \\
\hline Ítem 46 & 224.79 & 174.38 & .420 & .913 \\
\hline Ítem 47 & 225.08 & 171.11 & .404 & .913 \\
\hline Ítem 48 & 224.75 & 172.54 & .482 & .912 \\
\hline Ítem 49 & 225.07 & 168.76 & .654 & .910 \\
\hline Ítem 50 & 224.69 & 176.25 & .301 & .914 \\
\hline Ítem 51 & 224.66 & 175.20 & .443 & .912 \\
\hline Ítem 52 & 224.63 & 177.98 & .203 & .914 \\
\hline
\end{tabular}


María Ángeles Blanco Portillo, Paz Cánovas Leonhardt, Piedad Sahuquillo Mateo, Amparo Pérez Carbonell y Verónica Riquelme Soto

\begin{tabular}{lcccc}
\hline & $\begin{array}{c}\text { Media de } \\
\text { escala si el } \\
\text { elemento se } \\
\text { ha suprimido }\end{array}$ & $\begin{array}{c}\text { Varianza de } \\
\text { escala si el } \\
\text { elemento se ha } \\
\text { suprimido }\end{array}$ & $\begin{array}{c}\text { Correlación } \\
\text { total de } \\
\text { elementos } \\
\text { corregida }\end{array}$ & $\begin{array}{c}\text { Alfa de } \\
\text { Cronbach si el } \\
\text { elemento se } \\
\text { ha suprimido }\end{array}$ \\
\hline Ítem 53 & 224.84 & 175.13 & .328 & .913 \\
Ítem 54 & 224.98 & 171.35 & .569 & .911 \\
Ítem 55 & 225.03 & 172.84 & .448 & .912 \\
Ítem 56 & 224.61 & 175.48 & .492 & .912 \\
Ítem 57 & 225.18 & 170.84 & .555 & .911 \\
Ítem 58 & 224.94 & 171.35 & .586 & .911 \\
Ítem 59 & 224.83 & 172.22 & .574 & .911 \\
Ítem 60 & 224.71 & 174.71 & .448 & .912 \\
Ítem 61 & 224.88 & 174.18 & .415 & .913 \\
Ítem 62 & 224.98 & 175.73 & .249 & .915 \\
Ítem 63 & 224.89 & 172.60 & .512 & .912 \\
Ítem 64 & 225.40 & 170.96 & .317 & .915 \\
Ítem 65 & 225.13 & 172.89 & .442 & .912 \\
Ítem 66 & 224.62 & 176.51 & .377 & .913 \\
Ítem 67 & 224.91 & 170.64 & .498 & .912 \\
Ítem 68 & 225.61 & 170.43 & .329 & .915 \\
\hline Fuente: Blant & & & &
\end{tabular}

Fuente: Blanco (2016).

\section{Análisis de resultados}

\section{Análisis factorial exploratorio}

Para conocer el agrupamiento de los ítems en diversos factores se realizó un análisis factorial exploratorio inicial a partir de los 42 ítems. Dicho análisis se llevó a cabo bajo el método de extracción de componentes principales y posterior rotación Oblimin, con el cálculo previo al análisis, de la medida de adecuación muestral de Kaiser-Meyer-Olkin (KMO) y el test de esfericidad de Bartlett. La puntuación fijada para establecer el número de factores parte de un autovalor fue $>$ de 1, aplicándose esta a cada constructo. De esta forma, se eliminaron aquellos ítems con valores factoriales inferiores a 0,30 .

En este sentido, un primer análisis factorial exploratorio con los 42 ítems permitió reducir la escala a diez factores, con autovalores mayores que uno, permitiendo explicar un $54,60 \%$ de la varianza. El índice KMO evidenció un valor de ,894 y el test de Bartlett resultó estadísticamente 
significativo $(p<, 001)$. Pese a ello, se eliminó el ítem número 65 "me considero un buen/a padre/madre" cuya carga factorial resultó inferior a 0,3 .

Tras llevar a cabo dicho ajuste, se realizó un segundo análisis factorial que permitió extraer once factores a partir de los 41 ítems restantes, explicando el $57,92 \%$ de la varianza total. El primero de ellos satisface por sí mismo el $25,14 \%$ del total explicado. El resto de los factores presentan explicaciones que se encuentran entre un 7,46\% (corresponde al segundo factor), hasta un 4,21\% (correspondiente al factor 11). Así pues, se concibe que en términos generales todas las dimensiones/factores presentan un suficiente un nivel de explicación de varianza, como se observa en la tabla 4.

El índice KMO del segundo análisis señaló un valor de ,897 y el test de Bartlett fue estadísticamente significativo $(p<, 001)$. Dichos resultados, propios del análisis factorial exploratorio, se encuentran reflejados en la tabla 5. A su vez, la fiabilidad para el conjunto de la escala reflejó un alfa de Cronbach de ,92, mostrando este una elevada consistencia interna.

Tabla 4

Contribuciones (\%) a la explicación de la varianza (total y explicada) por los factores resultantes

\begin{tabular}{ccccc}
\hline FACTOR & 1 & 2 & 3 & 4 \\
\hline 1 & 25.14 & 25.14 & 43.40 & 43.40 \\
2 & 4.32 & 29.46 & 7.46 & 50.86 \\
3 & 4.02 & 33.48 & 6.94 & 57.80 \\
4 & 3.83 & 37.31 & 6.61 & 64.41 \\
5 & 3.59 & 40.90 & 6.20 & 70.61 \\
6 & 3.38 & 44.28 & 5.83 & 76.44 \\
7 & 3.01 & 47.29 & 5.20 & 81.64 \\
8 & 2.90 & 50.19 & 5.01 & 86.65 \\
9 & 2.68 & 52.87 & 4.63 & 91.28 \\
10 & 2.59 & 55.46 & 4.47 & 95.75 \\
11 & 2.46 & 57.92 & 4.21 & 100.00 \\
\hline
\end{tabular}

Nota. 1. Explicación de la varianza total. 2. Explicación acumulada de la varianza total. 3. Varianza explicada en el espacio n-factorial. 4. Varianza explicada acumulada en el espacio n-factorial.

Fuente: Blanco (2016). 
La Competencia Parental en las etapas de Educación Infantil y Primaria: Diseño y Validación de un Instrumento

María Ángeles Blanco Portillo, Paz Cánovas Leonhardt, Piedad Sahuquillo Mateo, Amparo Pérez Carbonell y Verónica Riquelme Soto

A continuación, en la tabla 5, se muestran aquellos ítems que componen cada factor, al igual que la varianza explicada para cada una de las dimensiones.

Tabla 5

Análisis factorial exploratorio de la escala de competencia parental

\begin{tabular}{|c|c|c|c|c|c|c|c|c|c|c|c|c|}
\hline & F1 & F2 & F3 & F4 & F5 & F6 & F7 & F8 & F9 & F10 & F11 & Com. \\
\hline \multicolumn{13}{|l|}{ Factor 1} \\
\hline Ítem 58 & .694 & .033 & .025 & -.153 & -.033 & .162 & -.069 & -.081 & .096 & .064 & .058 & .653 \\
\hline Ítem 54 & .623 & -.067 & -.008 & .053 & -.084 & .148 & -.010 & .163 & .122 & .054 & -.044 & .609 \\
\hline Ítem 23 & .598 & .129 & -.077 & -.062 & .084 & .025 & .188 & .083 & -.066 & -.063 & -.025 & .493 \\
\hline Ítem 22 & .530 & -.104 & -.055 & .022 & .113 & .049 & -.062 & .095 & .041 & -.315 & .214 & .533 \\
\hline Ítem 57 & .526 & -.086 & .146 & -.106 & .004 & .069 & .235 & -.060 & .063 & .120 & .068 & .527 \\
\hline Ítem 45 & .425 & .321 & .134 & .116 & -.082 & -.172 & -.086 & .171 & .183 & .071 & -.048 & .514 \\
\hline Ítem 56 & .398 & .003 & .122 & -.041 & -.036 & .052 & .002 & .190 & -.063 & -.152 & .125 & .423 \\
\hline \multicolumn{13}{|l|}{ Factor 2} \\
\hline Ítem 21 & .032 & .749 & .022 & .023 & -.013 & .036 & .078 & .209 & -.153 & -.046 & .140 & .684 \\
\hline Ítem 31 & -.056 & .355 & -.045 & -.245 & -.222 & .172 & -.069 & .031 & .281 & -.169 & -.029 & .531 \\
\hline \multicolumn{13}{|l|}{ Factor 3} \\
\hline Ítem 50 & -.084 & .136 & .868 & .028 & .025 & .039 & .070 & .025 & -.174 & .070 & -.121 & .746 \\
\hline Ítem 51 & .035 & -.253 & .725 & -.170 & .028 & .044 & -.019 & .009 & .083 & -.063 & .081 & .677 \\
\hline \multicolumn{13}{|l|}{ Factor 4} \\
\hline Ítem 61 & -.057 & -.100 & .046 & -.722 & -.121 & .043 & .037 & .147 & .030 & .042 & .048 & .641 \\
\hline Ítem 60 & .159 & .048 & .121 & -.643 & -.043 & -.035 & .038 & -.041 & .116 & -.042 & .003 & .586 \\
\hline Ítem 59 & .064 & .132 & .228 & -.314 & -.124 & .261 & -.155 & .028 & .204 & -.050 & .091 & .544 \\
\hline \multicolumn{13}{|l|}{ Factor 5} \\
\hline Ítem 26 & .003 & .079 & -.091 & -.097 & -.720 & .044 & -.045 & -.041 & .009 & -.036 & .111 & .581 \\
\hline Ítem 25 & -.050 & .012 & .062 & -.081 & -.713 & .057 & .020 & -.003 & .048 & -.011 & .031 & .589 \\
\hline Ítem 27 & .040 & -.168 & .241 & .378 & -.406 & -.127 & .069 & .247 & .069 & -.104 & .156 & .568 \\
\hline Ítem 24 & .379 & .113 & .103 & -.074 & -.392 & -.052 & .125 & .012 & -.063 & -.175 & -.145 & .557 \\
\hline \multicolumn{13}{|l|}{ Factor 6} \\
\hline Ítem 48 & .144 & .029 & .020 & -.063 & .121 & .752 & .055 & .058 & -.007 & -.022 & -.059 & .667 \\
\hline Ítem 47 & .037 & -.050 & -.008 & .004 & -.101 & .727 & .045 & .002 & -.030 & -.070 & -.041 & .588 \\
\hline Ítem 38 & -.121 & .157 & .169 & .089 & .059 & .460 & .034 & .063 & .197 & .286 & .216 & .499 \\
\hline \multicolumn{13}{|l|}{ Factor 7} \\
\hline Ítem 37 & .028 & .138 & .063 & .023 & ,054 & .116 & 679 & -.053 & .035 & .069 & .078 & .567 \\
\hline Ítem 36 & .033 & -.108 & .108 & .040 & .195 & .008 & .609 & .031 & .220 & -.248 & .044 & .580 \\
\hline Ítem 42 & .040 & .002 & -.065 & -.172 & -.303 & .046 & .519 & .014 & -.072 & .297 & .085 & .559 \\
\hline Ítem 46 & .073 & -.001 & .083 & -.159 & -.242 & -.006 & .382 & .172 & -.006 & -.125 & -.311 & .497 \\
\hline \multicolumn{13}{|l|}{ Factor 8} \\
\hline Ítem 35 & .043 & .148 & .061 & -.188 & .216 & -.050 & -.071 & .719 & .094 & .003 & .060 & .657 \\
\hline Ítem 33 & -.044 & .039 & -.013 & .073 & -.093 & .263 & .108 & .626 & -.025 & -.103 & -.129 & .588 \\
\hline Ítem 44 & .179 & .148 & .156 & -.025 & -.061 & -.014 & -.107 & .568 & .094 & .125 & -.058 & .570 \\
\hline Ítem 34 & .034 & -.039 & -.064 & .082 & -.051 & .154 & .356 & .526 & .101 & -.126 & .050 & .642 \\
\hline
\end{tabular}


La Competencia Parental en las etapas de Educación Infantil y Primaria: Diseño y Validación de un Instrumento

María Ángeles Blanco Portillo, Paz Cánovas Leonhardt, Piedad Sahuquillo Mateo, Amparo Pérez Carbonell y Verónica Riquelme Soto

\begin{tabular}{|c|c|c|c|c|c|c|c|c|c|c|c|c|}
\hline & F1 & F2 & F3 & F4 & F5 & F6 & F7 & F8 & F9 & F10 & F11 & Com. \\
\hline \multicolumn{13}{|l|}{ Factor 9} \\
\hline Ítem 43 & -.048 & -.172 & -.127 & -.164 & .093 & .009 & .075 & .191 & .709 & -.036 & .031 & .607 \\
\hline Ítem 29 & .302 & .050 & .140 & .060 & -.110 & -.002 & .090 & -.070 & .579 & .098 & -.145 & .618 \\
\hline Ítem 30 & .074 & .329 & -.081 & -.096 & -.221 & .111 & .132 & -.055 & .456 & -.086 & -.063 & .613 \\
\hline \multicolumn{13}{|l|}{ Factor 10} \\
\hline Ítem 28 & .206 & .106 & -.081 & .031 & -.252 & .047 & .073 & .191 & -.019 & -.491 & -.001 & .576 \\
\hline Ítem 32 & -.085 & .058 & .158 & -.013 & -.301 & .099 & .028 & .016 & .194 & -.453 & .030 & .505 \\
\hline Ítem 55 & .252 & -.123 & .156 & .114 & -.270 & -.051 & .067 & .172 & .203 & .370 & .189 & .561 \\
\hline \multicolumn{13}{|l|}{ Factor 11} \\
\hline Ítem 66 & -.008 & .222 & .001 & -.059 & -.126 & -.134 & .308 & -.033 & -.008 & .065 & .636 & .619 \\
\hline Ítem 53 & .221 & -.225 & -.013 & -.060 & -.110 & .283 & -.063 & .029 & -.168 & -.012 & .492 & .541 \\
\hline Ítem 63 & .095 & -.156 & -.043 & -.252 & -.167 & -.048 & -.054 & .387 & -.046 & .010 & .454 & .607 \\
\hline Ítem 20 & .039 & .120 & .179 & .128 & -.136 & .160 & .002 & -.139 & .240 & -.171 & .371 & .457 \\
\hline Ítem 67 & .108 & .162 & .318 & -.232 & .157 & -.111 & .089 & .018 & .019 & -.282 & .339 & .542 \\
\hline Autovalor & 4.38 & & & & & & & & & & & \\
\hline $\begin{array}{l}\text { Varianza ex- } \\
\text { plicada (\%) }\end{array}$ & 25.14 & 4.32 & 4.02 & 3.83 & 3.59 & 3.38 & 3.01 & 2.90 & 2.68 & 2.59 & 2.44 & \\
\hline $\begin{array}{l}\text { Número de } \\
\text { ítems }\end{array}$ & 7 & 2 & 2 & 3 & 4 & 4 & 4 & 4 & 3 & 3 & 5 & \\
\hline
\end{tabular}

Nota. Com.=Comunalidad; Método de extracción: Análisis de componentes principales; Método de rotación: Normalización Oblimin con Kaiser; La rotación ha convergido en 41 iteraciones.

Fuente: Blanco (2016).

\section{Análisis factorial confirmatorio}

La validez interna de la escala se analizó mediante la aplicación de diversos análisis factoriales confirmatorios que ratificaron la estructura factorial propuesta. La estimación del modelo se desarrolló a través del método Robusto de Estimación de Máxima Verosimilitud (MLR) a fin de corregir la falta de normalidad multivariante, mediante la utilización de estadísticos como el X2 de Satorra Bentler (Chou, Bentler y Satorra, 1991). Se calculó el coeficiente de Mardia a causa de la ausencia de normalidad, advirtiendo que superaba el punto de corte de 5 (511.01).

El modelo con los 41 ítems 5 iniciales distribuidos en los once factores propuestos por el análisis factorial exploratorio, no mostró un buen ajuste, tal y como se señala en la tabla 5. Con el fin de mejorar la estimación del modelo, se suprimieron los ítems que indicaban una carga factorial reducida y valores residuales estandarizados elevados $(<, 20)$.

5 Recordar al lector que en el análisis factorial exploratorio se descartó un ítem al presentar una carga factorial inferior a 0,3. 
Asimismo, se suprimieron los factores 2, 7, 10 y 11, ya que los ítems presentaban cargas factoriales reducidas $(<0,5)$. En relación a ello, se eliminaron los siguientes ítems del resto de factores:

- Factor 1: ítems 22 y 45.

- Factor 5: ítem 27.

- Factor 6: ítem 38.

- Factor 9: ítem 43.

De este modo, el modelo que mejor se ajusta a los datos recabados se integra de 22 ítems agrupados en siete dimensiones:

- Factor 1 de aceptación de la individualidad del menor, compuesto por los ítems 22, 54, 56, 57 y 58.

- Factor 3 de tiempo compartido en familia, está integrado por los ítems 50 y 51 .

- Factor 4 de responsabilidad y percepción parental formado por los ítems 59, 60 y 61 .

- Factor 5 de transmisión de valores, recoge la información contenida en los ítems 24, 25 y 26.

- Factor 6 apoyos hacia el menor, compuesto de los ítems 47, 48 y 49.

- Factor 8 de actitudes parentales positivas integrado por los ítems $33,34,35$ y 44.

- Factor 9 de comunicación y expresión afectiva, integrado por los ítems 29 y 30.

En la tabla 6, se presentan los principales indicadores de bondad de ajuste para determinar el ajuste de los AFC para la muestra consultada. El modelo definitivo presenta un chi-cuadrado significativo (S-B $2=$ 270.37; $d f=188 ; p<, 01)$ y un valor del chi-cuadrado normalizado $(2 / \mathrm{df}$ $=1,90$ ) que señala un buen ajuste, debido a que obtiene una puntuación inferior a cinco (Bollen, 1989; Byrne, 1989; Carmines y Mclver, 1981).

El Error de Aproximación Cuadrático Medio (RMSEA) obtuvo un valor de ,034, cumpliendo así con los criterios mínimos de ajuste aceptable (igual o inferior a 0,08; Browne y Cudeck, 1993).

Con ello, el resto de índices señalan un buen ajuste del modelo, ya que los valores son superiores a ,90 (Maccallum y Austin, 2000): el Índice de Ajuste No Normado (NNFI = ,94), el Índice de Ajuste Comparado $(\mathrm{CFI}=, 95)$ y el Índice de Ajuste Incremental $(\mathrm{IFI}=, 95)$. 
La Competencia Parental en las etapas de Educación Infantil y Primaria: Diseño y Validación de un Instrumento

María Ángeles Blanco Portillo, Paz Cánovas Leonhardt, Piedad Sahuquillo Mateo, amparo Pérez Carbonell y Verónica Riquelme Soto

Tabla 6

Índices de bondad de ajuste modelos

\begin{tabular}{|c|c|c|c|c|c|c|c|}
\hline Modelo & S-BX ${ }^{2}$ & gl & $\mathrm{X}^{2} / g \mid$ & RMSEA (IC) & $\mathrm{CFI}$ & $\mathrm{NNFI}$ & $\mathrm{IFI}$ \\
\hline 11 factores, 41 ítems & 1002.49 & 724 & 1,81 & $\begin{array}{c}.033 \\
(.028-.038)\end{array}$ & .89 & .88 & .90 \\
\hline 10 factores, 38 ítems & 864.97 & 620 & 1,83 & $\begin{array}{c}.033 \\
(.028-.038)\end{array}$ & .90 & .89 & .90 \\
\hline 9 factores, 34 ítems & 695.27 & 491 & 1,88 & $\begin{array}{c}.034 \\
(.028-.040)\end{array}$ & .91 & .89 & .91 \\
\hline 8 factores, 32 ítems & 608.46 & 436 & 1,85 & $\begin{array}{c}033 \\
(.027-.039)\end{array}$ & .92 & .91 & .92 \\
\hline 7 factores, 27 ítems & 424.26 & 303 & 1.82 & $\begin{array}{c}033 \\
(.025-.040)\end{array}$ & .94 & .93 & .94 \\
\hline 7 factores, 22 ítems & 270.37 & 188 & 1.90 & $\begin{array}{c}034 \\
(.024-.043)\end{array}$ & .95 & .94 & .95 \\
\hline
\end{tabular}

Nota. $\mathrm{gl}=$ grados de libertad; S-B=Satorra Bentler; RMSEA = Error de Aproximación Cuadrático Medio; IC=Intervalo de confianza del RMSEA; CFI = Índice de Ajuste Comparado; NNFI = Índice de Ajuste No Normado; IFI = Índice de Ajuste Incremental. Fuente: Blanco (2016).

En último lugar, la validez de constructo se contrastó a través del análisis de la validez convergente y discriminante. Por un lado, la validez convergente se mostró adecuada, ya que los ítems de la escala se mantienen significativamente correlacionados con las variables latentes que pretenden medir. En todos los casos los valores de t para las variables oscilaron desde 5,10 hasta 10,44 ( $\mathrm{t}>1,96)$, siendo estos significativos en el nivel de, 05 . Por otro lado, las cargas de cada factor promedio son superiores a ,60 (Kline, 2005) en la mayoría de indicadores, a excepción de los ítems que se presentan a continuación: el ítem $23(\lambda=, 54)$, el ítem $56(\lambda=, 58)$, el ítem $50(\lambda=, 51)$, el ítem $61(\lambda=, 56)$, el ítem $26(\lambda=$ ,56), el ítem $47(\lambda=, 54)$ y el ítem $35(\lambda=, 59)$, pese a que en la mayoría de casos presentan valores cercanos a , 60 .

Tabla 7

Resultados del análisis factorial confirmatorio

\begin{tabular}{llll}
\hline Ítems Factor & $\begin{array}{c}\text { Carga } \\
\text { factorial }\end{array}$ & a FC AVE \\
\hline & Factor 1. Aceptación de la individualidad del menor & .75 .77 .40 \\
\hline 23 & Le hago ver sus habilidades más que sus debilidades. & .54 & \\
54 & $\begin{array}{l}\text { Intento ponerme en el lugar del/a niño/a y entender } \\
\text { cómo se puede sentir. }\end{array}$ & .70 &
\end{tabular}


La Competencia Parental en las etapas de Educación Infantil y Primaria: Diseño y Validación de un Instrumento

María Ángeles Blanco Portillo, Paz Cánovas Leonhardt, Piedad Sahuquillo Mateo, Amparo Pérez Carbonell y Verónica Riquelme Soto

Ítems Factor

Carga factorial

a FC AVE

56 Valoro su esfuerzo.

.58

57

Acepto las cosas que les gustan aunque no me gusten a mí.

.63

58 Me adapto a él/ella cuando la situación lo requiere. $\quad .71$ Factor 3. Tiempo compartido en familia .62 .68 .54

50 Celebramos en familia todos los acontecimientos importantes.

51 Compartimos el tiempo que podemos en familia.

.90

Factor 4. Responsabilidad y percepción parental

59 Me pongo al día sobre los temas de interés que le afectan.

.67 .68 .41

60 Soy consciente de que las necesidades que tiene cambian según crece.

Soy consciente de mis fallos y fortalezas como

61 padre/madre.

.65

.56

Factor 5. Transmisión de valores

24 Induzco al/a niño/a a buscar soluciones ante una dificultad.

25 Le doy tareas a razón de su edad y capacidad.

26 Le motivo para que haga sus cosas sin mi ayuda. Factor 6. Apoyos hacia el menor

47 Pido y busco ayuda externa cuando él/ella lo necesita.

48 Le enseño a que busque ayuda cuando lo necesite. Le muestro diferentes formas de alcanzar lo que

49 quiere

Factor 8. Actitudes parentales positivas

33 Le felicito cada vez que hace una cosa bien.

.73 .74 .42

34 Le apoyo cuando me necesita.

35 Le animo en todo aquello que le gusta.

$44 \quad$ Le escucho cuando necesita contarme algo. Factor 9. Comunicación y expresión afectiva .66

.60

.56

.54

.61

.76

.66 .64 .37

\section{6}

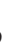
.66 .68 .41

\section{4}


La fiabilidad de la estructura factorial definitiva se realizó mediante el uso de tres indicadores, tal y como se muestra en la tabla 7 , siendo estos: el alfa de Cronbach, fiabilidad compuesta (FC) y la varianza media extraída (AVE).

Como se observa en la tabla 7, tanto los coeficientes del alfa de Cronbach, como los de la FC para cada factor, se encuentran entre ,62 y,77, siendo superiores o próximos al criterio recomendado (>,70; Hair, Black, Babin, Anderson y Tatham, 2006). Sin embargo, los valores de la AVE oscilaron entre .37 y .54, siendo solo superior en el factor 2 al punto de corte recomendado (>,50; Fornell y Larcker, 1981).

Tabla 8

Matriz de correlaciones interfactorial de la escala de competencia parental

\begin{tabular}{llllllll}
\hline & F1 & F3 & F4 & F5 & F6 & F8 & F9 \\
\hline F1 & 0.63 & & & & & & \\
F3 & $.304^{* *}$ & 0.73 & & & & & \\
F4 & $.504^{* *}$ & $.319^{* *}$ & 0.64 & & & & \\
F5 & $.458^{* *}$ & $.208^{* *}$ & $.399^{* *}$ & 0.61 & & & \\
F6 & $.542^{* *}$ & $.225^{* *}$ & $.457^{* *}$ & $.420^{* *}$ & 0.64 & & \\
F8 & $.535^{* *}$ & $.288^{* *}$ & $.396^{* *}$ & $.384^{* *}$ & $.459^{* *}$ & 0.65 & \\
F9 & $.477^{* *}$ & $.248^{* *}$ & $.384^{* *}$ & $.451^{* *}$ & $.374^{* *}$ & $.367^{* *}$ & 0.69 \\
\hline
\end{tabular}

Nota. ${ }^{* *} \mathrm{p} \leq 0,01$; en la diagonal se muestran los valores de la raíz del AVE para cada factor. Fuente: Blanco (2016).

La validez discriminante fue comprobada a partir de las correlaciones entre los diferentes factores inferiores a ,85 (Kline, 2005), cumpliéndose dicho criterio, tal y como se muestra en la tabla 8. Otro de los criterios empleados fue comprobar si la raíz cuadrada del AVE era superior a la correlación entre pares de factores (Fornell y Larcker, 1981), cumpliéndose dicho criterio para todas las dimensiones (ver tabla 7). Todo ello evidencia que los resultados presentan una adecuada validez discriminante.

\section{Conclusiones}

A partir de los resultados obtenidos, se evidencia la pertinencia de haber incluido en nuestro estudio dos dimensiones altamente relevantes, como son el apego y el ejercicio parental desde la responsividad. Sin 
María Ángeles Blanco Portillo, Paz Cánovas Leonhardt, Piedad Sahuquillo Mateo, Amparo Pérez Carbonell y Verónica Riquelme Soto

duda, esto ha permitido analizar nuestro objeto de estudio desde un enfoque más global e integrador, al tiempo que ha posibilitado superar carencias detectadas en otros instrumentos. En este sentido, el diseño del instrumento responde a la inexistencia de otros que permitiesen realizar un análisis exhaustivo desde la perspectiva epistemológica en que se enmarca nuestro trabajo.

Son muchos los instrumentos centrados en las debilidades de los padres y madres, al igual que en el grado de riesgo que presenta la infancia y adolescencia en relación a la educación que puedan estar recibiendo por parte de algunos adultos; no dando respuesta a la complejidad de la competencia parental. Cabe señalar que gran parte de dichas herramientas son de ámbito internacional, señalando que cuando estas han sido adaptadas a nuestro país han evidenciado limitaciones de diversa índole, metodológicas, psicométricas, entre otras.

El análisis de fiabilidad y validez del instrumento se realizó a partir de un análisis métrico la calidad técnica del mismo, afirmando que la validez resulta adecuada para la población estudiada, pero la fiabilidad compuesta y la varianza extraída de algunos factores/dimensiones presenta ciertos indicadores por debajo de 0,7 y 0,5. Es por ello que algunos factores no mostraron una adecuada fiabilidad.

Con el objeto de mejorar la estimación del modelo realizado, se eliminaron los factores con cargas factoriales reducidas, obteniendo 22 ítems agrupados en siete dimensiones. Por otra parte, se ha analizado la validez de constructo a través del análisis de la validez convergente y discriminante. La validez convergente fue significativa a nivel de ,05 y la validez discriminante ha confirmado que todas las dimensiones tenían valores inferiores a ,85 por lo que presenta una adecuada validez discriminante.

Atendiendo a los análisis realizados, podemos concluir que la contribución de nuestro trabajo es positiva para valorar la competencia parental en las etapas de Educación Infantil y Primaria, si bien hemos de reconocer las limitaciones del instrumento en cuanto a su aplicación en otras familias cuyos hijos se encuentren en otro nivel educativo. Es nuestro propósito continuar profundizando en esta línea con el fin de crear instrumentos adaptados a realidades concretas. 
La Competencia Parental en las etapas de Educación Infantil y Primaria: Diseño y Validación de un Instrumento

María Ángeles Blanco Portillo, Paz Cánovas Leonhardt, Piedad Sahuquillo Mateo, amparo Pérez Carbonell y Verónica Riquelme Soto

\section{Referencias}

Barudy, J. y Dantagnan, M. (2005). Los buenos tratos en la infancia. Parentalidad, apego y resiliencia. Barcelona: Gedisa.

Barudy, J. y Dantagnan, M. (2010). Los desafíos invisibles de ser madre o padre. Manual de evaluación de competencias y resiliencia parental. Barcelona: Gedisa.

Bayot, A., Hernández, J.V. y De Julián, L.F. (2005). Análisis factorial exploratorio y propiedades psicométricas de la escala de competencia parental percibida. Versión para padres/madres (ECPP-p). Relieve, 2, 113-126. Recuperado de: https://bit.ly/2CEYkX0

Bisquerra, R. y Pérez, N. (2007). Las competencias emocionales. Educación XXI: Revista de la Facultad de Educación, 10, 61-82. Recuperado de: https://bit.ly/1tkJ8GV

Blanco, M.A. (2016). Análisis de la competencia parental en las etapas de educación infantil y primaria: diseño de un instrumento. Tesis Doctoral. Universidad de Valencia.

Bollen, K. A. (1989). Structural equations with latent variables. New York: John Wiley \& Sons.

Browne, M. W. y Cudeck, R. (1993). Alternative ways of assessing model fit. In K. A. Byrne, B. (1989). A primer of LISREL: Basic applications and programming for confirmatory factor analytic models. New York: Springer-Verlag Publishing.

Cánovas, P. \& Sahuquillo, P. (2011). El desarrollo de competencias parentales como factor de resiliencia. XII Congreso Internacional de Teoría de la Educación. Barcelona. Recuperado de: http://www.cite2011.com/Comunicaciones/Familias/122.pdf

Carmines, E. y Mclver, J. (1981). Analyzing models with unobserved variables: Analysis of covariance structures. In Bohrnstedt, G. and Borgatta, E. (Eds.). Social Measurement: Current Issues (65-115). Beverly Hills, CA: Sage Publications.

Chou, C.P., Bentler, P.M. y Satorra, A. (1991). Scaled test statistics and robust standard errors for non-normal data in covariance structure analysis: a Monte Carlo study. British Journal of Mathematical and Statistical Psychology, 2, 347-357. Recuperado de: https://bit.ly/2TBzH3a

Díaz, J.V. (1993). Teoría de los tests. Psicometría II: clásica y de las respuestas al ítem. Valencia: Universidad de Valencia.

Fornell, C., y Larcker, D. F. (1981). Evaluating structural equation models with unobservable variables and measurement error. Journal of Marketing Research, 1, 39-50. Recuperado de: https://bit.ly/2JEbGZh

George, D. y Mallery, P. (1995). SPSS/PC + Step by: A Simple Guide and Reference. Belmont: Wadsworth Publishing Company.

Gronlund, N.E. y Linn, R.L. (1990). Student Exercise Manual for Measurement and Evaluation in Teaching. New York: Macmillan.

Hair, J.F., Black, W.C., Babin, B.J., Anderson, R.E., \& Tatham, R.L. (2006). Multivariate Data Analysis. New Jersey: Pearson.

Jornet, J.M., Suárez, J.M. y Pérez, A. (2000). La validez en la evaluación de programas. Revista de Investigación Educativa, 2, 341-356. Recuperado de: https://bit.ly/2Wm182q

Kline, R. B. (2005). Principles and practice of structural equation modeling. New York: The Guilford Press.

Mac Callum, R. y Austin, J. (2000). Applications of Structural Equation Modeling in 
La Competencia Parental en las etapas de Educación Infantil y Primaria: Diseño y Validación de un Instrumento

María Ángeles Blanco Portillo, Paz Cánovas Leonhardt, Piedad Sahuquillo Mateo, Amparo Pérez Carbonell y Verónica Riquelme Soto

Psychological Research. Annual Review of Psychology. 51, 201-226. Recuperado de: https://bit.ly/2utOTF8

Masten, A.S. y Curtis, W.J. (2000). Integrating competence and psychopathology: Pathways toward a comprehensive science of adaption undevelopment. Development and Psychopathology, 3, 529-550. Recuperado de: https://bit.ly/2FzAAp3

Masten, A. y Obradovic, J. (2006). Competence and resilience in development. Annals of the New York Academy of Sciences, 1, 13-27. Recuperado de: https://bit.ly/2HX09lg

Pereda, S. y Berrocal, F. (2001). Técnicas de gestión de recursos humanos por competencias. Madrid: Editorial Centro de Estudios Ramón Areces.

Repetto, E. (2003). La competencia emocional e intervenciones para su desarrollo. En E. Repetto (Ed.). Modelos de Orientación e Intervención Psicopedagógica. Madrid: UNED.

Repetto, E., Pena M., Mudarra, M.J. y Uribarri, M. (2007). Orientación de las competencias socioemocionales de los alumnos de Educación Secundaria en contextos multiculturales. Electronic Journal of Research in educational Psychology, 5, 159-178. Recuperado de: https://bit.ly/2Wm5834

Rodrigo, M.J., Máiquez, M.L., Byrne, S., Rodríguez, B., Martín, J.C., Rodríguez, G. y Pérez, L. (2008). Programa Crecer Felices en Familia: programa de apoyo psicoeducativo para promover el desarrollo infantil. Valladolid: Junta de Castilla y León.

Rodrigo, M.J., Martin, J.C., Cabrera, E. y Máiquez, M.L. (2009). Las competencias parentales en contextos de riesgo psicosocial. Intervención Psicosocial, 2, 113-120. Recuperado de: https://bit.ly/2UW6wce

Roe, R. (2003). What makes a competent psychologist? European Psychologist, 3, 192202. Recuperado de: https://bit.ly/2U21tdp

Rutter, M. (2007). Resilience, competence and coping. Child Abuse and Neglect, 3, 205209. Recuperado de: https://bit.ly/2TxIPqi

Tejada, J. y Navío, A. (2004). El desarrollo y la gestión de las competencias profesionales: una mirada desde la formación. Revista Iberoamericana de Educación, 2, 1-15. Recuperado de: https://bit.ly/2HSVdhh

Tejada, J. (2005). El trabajo por competencias en el prácticum: cómo organizarlo y cómo evaluarlo. Revista Electrónica de Investigación Educativa, 2, 2-31. Recuperado de: https://bit.ly/2 HNdD3f

Vázquez, N., Molina, M.C., Ramos, P. y Artazcoz, L. (2016). Validación de un instrumento en español para medir habilidades parentales promovidas en una intervención de educación parental. Revista d'Innovació i Recerca en Educació, 2, 30-47. Recuperado de: https://bit.ly/2LzFwtM

Wilcox, M.J., Kauni, T. y Caswell, S. (1990). Partner sensitivity to communication behaviour of young children with developmental disabilities. Journal of Speech and Hearing Disorders, 4, 679-693. Recuperado de: https://bit.ly/2Fyf3Nw

Zabala, A. y Arnau, L. (2007). 11 ideas clave: como aprender y enseñar competencias. Grao: Barcelona. 
